\title{
Diagnóstico da utilização de sistema de informações geográficas na gestão do saneamento básico das regiões administrativas de Araçatuba e Bauru (sp)
}

\author{
Diagnostic of the use of geographic information system in the \\ management of the basic sanitation of the Administrative Regions of \\ Araçatuba and Bauru (SP)
}

\begin{abstract}
Enaldo Pires Montanha Júnior | Sergio Antonio Röhm
Data de entrada: 26/09/2012 | Data de aprovação: 14/02/2014

DOI: http://dx.doi.org/10.4322/dae.2014.122

Resumo

Os gestores de saneamento urbano no Brasil estão em busca de desenvolvimento, principalmente em termos de infraestrutura e tecnologia. $\mathrm{O}$ controle e o gerenciamento das informações geométricas e descritivas das redes de abastecimento de água e de esgoto sanitário são essenciais para tomadas de decisões eficientes e eficazes. A tecnologia de Sistema de Informações Geográficas vem sendo utilizada em diversos segmentos da administração urbana há algum tempo e em saneamento urbano não é diferente, pois a maioria dos grandes órgãos gestores do país já apresenta resultados convincentes de que o investimento nessa tecnologia traz vantagens técnicas e econômicas. O objetivo deste trabalho é diagnosticar, através de pesquisas de campo, a situação do uso de Sistemas de Informações Geográficas por órgãos vinculados ao município gestores de saneamento urbano das Regiões Administrativas de Araçatuba e de Bauru - SP, considerando sua utilização, instalações, dificuldades, principais motivos de não utilizar a tecnologia e as tendências de uso no futuro.
\end{abstract}

Palavras-chave: gestão; rede de abastecimento, rede de esgoto.

\begin{abstract}
The managers of urban sanitation in Brazil are seeking to development, especially in terms of infrastructure and technology. The control and management of geometric and descriptive information networks of water supply and sanitary sewer are essential for efficient and effective decision making. The technology of Geographic Information System has been used in several segments of the urban administration for some time and in urban sanitation is not different, as most of the major governing bodies in the country already shows convincing results that the investment in this technology brings technique and economic advantages. The objective of this work is to diagnose, by field research, the situation of the use of Geographic Information Systems to manage the urban sanitation by the agencies of the Administrative Regions of Araçatuba and Bauru - SP, considering its use, facilities, difficulties, major reasons not use technology and usage trends in the future.
\end{abstract}

Key-words: management, supply network, sewage network.

Enaldo Pires Montanha Júnior*

Mestre em Engenharia Urbana pela Universidade Federal de São Carlos - UFSCar. Supervisor de negócios do Centro de Tecnologias em Geoprocessamento - CTGEO

Sergio Antonio Röhm

Doutor em Geotecnia - Professor Associado do Departamento de Engenharia Civil e do Programa de Pós-graduação em Engenharia Urbana da UFSCar

*Endereço para correspondência: Centro de Tecnologias em Geoprocessamento - Av. Nicolau Zarvos, 1925 - CEP: 16401-371 - Lins-SP.

E-mail: enaldojr@ceteclins.com.br 


\section{Introdução}

No gerenciamento global de um sistema de abastecimento de água, auxiliados por sistemas de informações geográficas, denotam-se áreas de atividades específicas que manejam diferentes informações da base georreferenciada, tais como: i) sistema de informação de clientes; ii) projetos e planejamento; iii) operação e manutenção do sistema hidráulico (redes e dispositivos); e iv) gerências de infraestrutura, de fugas e administrativa. O conjunto geral destas informações disponibilizadas ao gestor permite definir as ações locais e globais de gerência do sistema. Outro aspecto relevante são as possibilidades de armazenamento e gestão da informação que são praticamente ilimitadas, citando como exemplos: i) visualização de todos os elementos da rede e de suas características; ii) gerações de estatísticas; iii) quantificação de número de válvulas de um determinado modelo e diâmetro; iv) gestão de consumidores e do parque de hidrômetros; e v) estado de funcionamento do sistema e etc. (DORCA; LUVIZOTTO; ANDRADE, 2001).

Os sistemas de abastecimento de água estão sujeitos a vários problemas, tais como: falta de restrição de uso de matéria prima, falta de recursos financeiros e descontinuidade administrativa. Para solucionar esses, e outros, é indispensável uma gestão eficiente nesses sistemas. A gestão de um sistema de abastecimento de água envolve o manuseio de grande quantidade de informações provenientes de fontes e naturezas distintas e disponibilizadas sob várias formas, tais como: planilhas, banco de dados, mapas, plantas de projeto, etc. (FERREIRA, 2005 e DORCA; LUVIZOTTO; ANDRADE, 2001).

Os sistemas de informações geográficas constituem ferramenta de gestão desejável considerando os benefícios administrativos, técnicos e econômicos. Entretanto, sua implantação efetiva é onerosa e um planejamento mal feito pode levar ao malogro todas as expectativas de benefícios que se objetivavam com a sua implantação. Além disso, os pequenos sistemas de abastecimentos de água que atendem às pequenas e médias cidades, de forma geral, não dispõem de corpo técnico qualificado e de recursos para a implantação adequada de um sistema de informações geográficas (FERREIRA, 2005).

Segundo Saiani (2007, p. 8):

o Brasil apresenta elevado déficit de acesso a serviços de saneamento básico. Devido aos impactos positivos desses serviços sobre o desenvolvimento econômico, é de fundamental importância que a universalização do acesso seja alcançada. No entanto, um conjunto de fatores restringe a expansão dos investimentos no setor: ausência de uma política clara, fragmentação de competências, ausência de uma regulação especifica, ineficiência de grande parte dos prestadores, forte presença pública no setor, fazendo com que os investimentos sejam inviabilizados pelos limites de endividamento, pelas metas de superávit e pelos contingenciamentos de créditos ao setor público.

Rocha; Vieira; Carneiro (2007) afirmam que a tecnologia de sistemas de informações geográficas permite, dentre outras ações, uma visão abrangente da distribuição de toda rede de abastecimento de água e esgoto sanitário, possibilitando identificar: i) falhas na base cartográfica; ii) os maiores consumidores e seus dados cadastrais; iii) as áreas de maior arrecadação; iv) os clientes inadimplentes; e v) as ligações cortadas, ligadas e suprimidas, dentre outras. Outras melhorias importantes são i) o acesso aos dados por todos departamentos da empresa, que repercute positivamente na execução, planejamento operacional, manutenção e atualização das informações em curto período de tempo, e ii) o uso e a distribuição otimizados de um bem público e finito como a água.

Considerando Ferreira (2005) e Rocha; Vieira; Carneiro (2007) pode-se concluir que a ineficiência de grande parte dos prestadores, citada por Saiani (2007), pode ser atenuada com o uso de sistemas de informações geográficas, visando a eficácia e a eficiência nas áreas administrativas, técnicas e econômicas dos sistemas de saneamento urbano.

As naturezas jurídico-administrativas assumidas pelos prestadores de serviços de saneamento básico existentes atualmente no Brasil são várias, destacando-se: secretarias, departamentos ou outros órgãos da administração pública direta; autarquia; empresa pública; sociedade de economia mista com gestão pública; sociedade de economia mista com gestão privada; e empresa privada e organização social (SAIANI, 2007).

Neste trabalho foram estudadas as Regiões Administrativas de Araçatuba e Bauru do Estado de São Paulo com a finalidade de entender, empregando pesquisas de campo, em que situação se encontra o uso de sistemas de informações geo- 
gráficas como auxiliar da gestão do saneamento urbano considerando os órgãos da administração pública direta.

Na Figura 1 observa-se um destaque dessas duas regiões administrativas. A Região Administrativa de Bauru está representada em vermelho e a de Araçatuba em azul. Nota-se que o conjunto de ambas recobre uma parte significativa do interior do Estado de São Paulo.

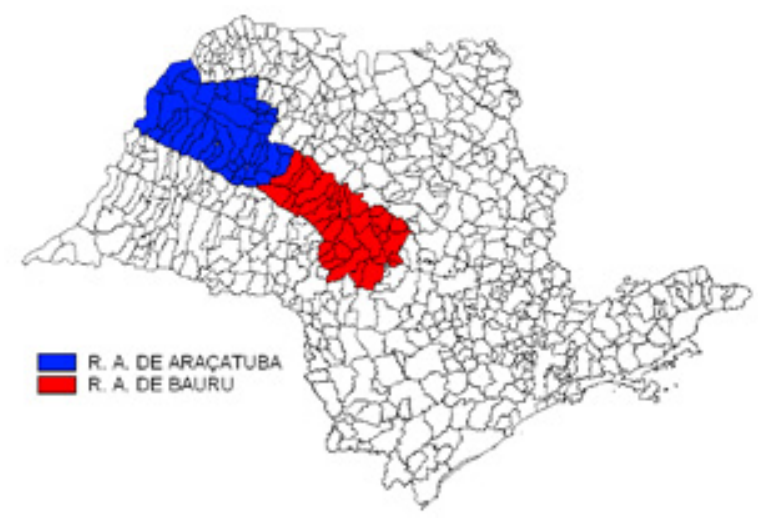

Figura 1 - Regiões Administrativas de Araçatuba-SP e Bauru-SP.

A Região Administrativa de Araçatuba é composta por 43 municípios: Andradina, Alto Alegre, Araçatuba, Auriflama, Avanhandava, Barbosa, Bento de Abreu, Bilac, Birigui, Braúna, Brejo Alegre, Buritama, Castilho, Clementina, Coroados, Gabriel Monteiro, Gastão Vidigal, General Salgado, Glicério, Guaraçaí, Guararapes, Guzolândia, Ilha Solteira, Lavínia, Lourdes, Luiziânia, Mirandópolis, Murutinga do Sul, Penápolis, Pereira Barreto, Piacatu, Nova Castilho, Nova Independência, Nova Luzitânia, Rubiácea, Santo Antonio do Aracanguá, Santópolis do Aguapeí, Sud Mennucci, Turiúba , Itapura, São João de Iracema, Suzanápolis e Valparaíso.

Os municípios mais populosos (no ano de 2011) são Araçatuba, com 182.474 habitantes, Birigui, com 109.944 habitantes, Penápolis, com 58.729 habitantes e Andradina, com 55.421 habitantes. Esses quatro municípios concentram mais de $54,85 \%$ do total da população regional (SEADE, 2014).

A Região Administrativa de Bauru é composta por 39 municípios: Agudos, Arealva, Areiópolis, Avaí, Balbinos, Barra Bonita, Bariri, Bauru, Bocaina, Boracéia, Borebi, Cabrália Paulista, Cafelândia, Dois Córregos, Duartina, Getulina, Guai- çara, Guaimbê, Guarantã, Iacanga, Igaraçu do Tietê, Jaú, Lençóis Paulista, Lins, Macatuba, Mineiros do Tietê, Paulistânia, Pederneiras, Pirajuí, Piratininga, Pongaí, Presidente Alves, Promissão, Reginópolis, Sabino, Ubirajara e Uru.

Os municípios de Bauru e Jaú são os dois únicos com população superior a 100.000 habitantes. Bauru possui 345.913 habitantes e Jaú possui 132.617 habitantes (no ano de 2011). Dentre todos os municípios da região, 27 possuem população inferior a 20 mil habitantes, sendo que 12 destes têm menos de 5.000 habitantes, abrigando menos de $4 \%$ da população total (SEADE, 2014).

\section{Objetivos}

O objetivo deste trabalho é diagnosticar, através de pesquisas de campo, a situação do uso de sistemas de informações geográficas por órgãos gestores da administração pública direta de saneamento urbano das Regiões Administrativas de Araçatuba e de Bauru - SP. Os aspectos considerados são a utilização, as facilidades, as dificuldades, os principais motivos de não utilizar a tecnologia e as tendências de uso no futuro.

\section{Métodos}

Os métodos de pesquisa utilizados foram baseados em questionários e visitas, que possibilitaram identificar quais são os municípios usuários de sistemas de informações geográficas e os que não são usuários para auxiliar na gestão dos sistemas de saneamento urbano.

Em seguida o grupo de usuários de sistemas de informações geográficas e grupo de não usuários foram reanalisados através de pesquisa do tipo Observação Direta Intensiva e Extensiva (MARCONI; LAKATOS, 2007), respectivamente.

Métodos para analisar o uso de sistemas de informações geográficas

Esta pesquisa, aplicada ao grupo de municípios usuários de sistemas de informações geográficas, foi do tipo "Observação Direta Intensiva" pela técnica de entrevista.

Os temas da entrevista foram: sistema de informações geográficas, base cartográfica e técnicas de implantação; estrutura física e humana; principais funções e instalações do sistema de informações geográficas implantado; setores e usuários da ferramenta; e nível de satisfação e perspectivas para o futuro. 
Métodos para analisar os motivos da não utilização de sistemas de informações geográficas

Nesta parte da pesquisa foi utilizado o método "Observação Direta Extensiva”, que pode ser realizada por meio de questionários, formulários, medidas de opinião e atitudes e de técnicas mercadológicas. Neste caso o método escolhido foi o questionário.

Os temas deste questionário foram:

i) formas de representação das redes abastecimento de água e de esgoto sanitário, com as opções: somente mapas analógicos, mapas analógicos e software $\mathrm{CAD}$, somente software $\mathrm{CAD}$, outros e não há representação;

ii) porcentagem da atualização da representação das redes abastecimento de água e de esgoto sanitário;

iii) formas de armazenamento das informações descritivas das redes abastecimento de água e de esgoto sanitário, com as opções: somente mapas analógicos, mapas analógicos e software CAD, banco de dados digital, planilhas eletrônicas, outros e não estão armazenadas; iv) conhecimento da existência do software de sistema de informações geográficas para gestão de redes abastecimento de água e de esgoto sanitário, com as seguintes opções: sim e não; e

v) principal motivo da ainda não aquisição do software de sistema de informações geográficas, com as seguintes opções: viabilidade técnica, problemas em recursos humanos, problemas com estrutura física, fatores econômicos, fatores políticos, outras prioridades, conhecimento há pouco tempo.

O objetivo desta fase foi conhecer a atual situação da gestão das informações das redes de abastecimento de água e de esgoto sanitário, identificar se os responsáveis conhecem a tecnologia de sistemas de informações geográficas, e por fim, saber o motivo de ainda não ter implantado esta no município.

\section{Resultados e discussões}

As Regiões Administrativas de Araçatuba e de Bauru somam 82 municípios dos quais 55 gerenciam o saneamento urbano (redes de abastecimento de água e de esgoto sanitário)

\begin{tabular}{|c|c|c|c|c|}
\hline \multirow{2}{*}{ Funções } & \multicolumn{4}{|c|}{ Municípios } \\
\hline & Município A & Município B & Município C & Município D \\
\hline Sistema de Inf. Geog. & SIG Integrado & SIG Integrado & SIG Dual & SIG Integrado \\
\hline Base Cartográfica & Completa & Completa & Completa & Incompleta \\
\hline Técnicas de Implantação & $\begin{array}{l}\text { Através de mapas } \\
\text { analógicos e conheci- } \\
\text { mentos de técnicos. }\end{array}$ & $\begin{array}{l}\text { Através de mapas } \\
\text { analógicos e conheci- } \\
\text { mentos de técnicos. }\end{array}$ & $\begin{array}{l}\text { Através de mapas } \\
\text { analógicos e } \\
\text { conhecimentos de } \\
\text { técnicos. }\end{array}$ & $\begin{array}{l}\text { Através de mapas } \\
\text { analógicos e conheci- } \\
\text { mentos de técnicos. }\end{array}$ \\
\hline Estrutura Física & Adequada & Adequada & Adequada & Inadequada \\
\hline Recursos Humanos & $\begin{array}{l}\text { Falta de treinamento } \\
\text { em SIG. }\end{array}$ & Adequadas & $\begin{array}{l}\text { Falta de treinamen- } \\
\text { to em SIG. }\end{array}$ & Inadequadas \\
\hline $\begin{array}{l}\text { Principais Funções e Vanta- } \\
\text { gens do SIG Implantado }\end{array}$ & $\begin{array}{l}\text { Gerenciamento de } \\
\text { informações }\end{array}$ & $\begin{array}{l}\text { Gerenciamento de } \\
\text { informações }\end{array}$ & $\begin{array}{l}\text { Consulta rápido ás } \\
\text { informações }\end{array}$ & $\begin{array}{l}\text { Gerenciamento de } \\
\text { informações }\end{array}$ \\
\hline $\begin{array}{l}\text { Setores e Usuários da Ferra- } \\
\text { menta }\end{array}$ & $\begin{array}{l}\text { Atualmente ninguém } \\
\text { utiliza. }\end{array}$ & $\begin{array}{l}\text { Diretoria, cadastro } \\
\text { e almoxarifado do } \\
\text { departamento e } \\
\text { secretaria de obras } \\
\text { da Prefeitura. }\end{array}$ & $\begin{array}{l}\text { Cadastro e geo- } \\
\text { processamento e } \\
\text { diretorias de produ- } \\
\text { ção, planejamento } \\
\text { e topografia no } \\
\text { departamento. }\end{array}$ & $\begin{array}{l}\text { Cadastro e diretoria do } \\
\text { departamento }\end{array}$ \\
\hline Nível de Satisfação & Indefinida & Ótima & Regular & Regular \\
\hline Perspectivas para o Futuro & $\begin{array}{l}\text { Atualização do SIG, } \\
\text { treinamento e inte- } \\
\text { grações com outros } \\
\text { sistemas. }\end{array}$ & $\begin{array}{l}\text { Integrações com } \\
\text { outros sistemas }\end{array}$ & $\begin{array}{l}\text { Aquisição de SIG } \\
\text { Integrado e em } \\
\text { integrações com } \\
\text { outros sistemas. }\end{array}$ & $\begin{array}{c}\text { Integrações com } \\
\text { outros sistemas e me- } \\
\text { lhorias em estruturas } \\
\text { física }\end{array}$ \\
\hline
\end{tabular}

Quadro 1 - Resumo das respostas dos municípios usuários de sistemas de informações geográficas. 
através da administração pública direta. Constatou-se que destes 55 municípios apenas 4 utilizam sistemas de informações geográficas para auxiliar na gestão das redes de abastecimento de água e de esgoto sanitário urbanas. Na Região Administrativa de Bauru identificaram-se 3 municípios usuários da tecnologia e na região de Araçatuba apenas 1.

Chega a ser surpreendente o pequeno número de usuários de sistemas de informações geográficas pelos órgãos gestores de saneamento nas regiões de Araçatuba e Bauru. Principalmente porque São Paulo é um dos Estados mais desenvolvidos tecnologicamente do Brasil e também com maiores recursos econômicos.

\section{Municípios usuários de sistemas de infor- mações geográficas}

O Quadro 1 resume os resultados das entrevistas nos 4 municípios usuários de sistemas de informações geográficas. Os itens de cor azul são considerados satisfatórios e de cor fúcsia os insatisfatórios na implantação e uso.

Como a quantidade de implantações é pequena, não se podem inferir conclusões expressivas ao estudar as regiões administrativas. Entretanto, algumas constatações podem ser discutidas.

As soluções mais utilizadas seguem a tendência da evolução tecnológica, mostrando que $75 \%$ dos municípios usuários de sistemas de informações geográficas deram preferência por softwares com arquitetura integrada (Câmara; Queiroz, 2012). Portanto, há apenas um usuário de sistema de informações geográficas de arquitetura dual (Câmara; Queiroz, 2012).

Outro aspecto importante é que dos quatro municípios usuários de sistemas de informações geográficas, apenas um (município D) ainda não possuía base cartográfica completa na época da conclusão destas análises. Este município foi o único que não terceirizou os serviços de elaboração da base cartográfica.

As informações cartográficas foram implantadas no banco de dados digital a partir de mapas existentes na Prefeitura Municipal, associados aos conhecimentos de técnicos relacionados com o saneamento urbano.

A estrutura física, de forma geral, foi considerada adequada (75\%) com uma ocorrência inadequada (25\%).
Os recursos humanos fragilizam as implantações analisadas. Apenas um município (25\%) admitiu que os recursos humanos estavam adequados às necessidades operacionais do sistema de informações geográficas. Os demais (75\%) têm consciência de que os recursos humanos são inadequados ou sem conhecimento sobre o assunto.

Embora os recursos humanos sejam, em sua maioria, despreparados para trabalhar com essa tecnologia, a maioria dos municípios entende que os sistemas de informações geográficas são vantajosos para auxiliar na gerência das informações (75\%). Um município valorizou apenas o acesso rápido às informações, minimizando o potencial do sistema de informações geográficas como instrumento fundamental para auxiliar nas tomadas de decisões técnicas, econômicas e administrativas.

O município A não estava usando a tecnologia implantada na época da aplicação da entrevista. $\mathrm{O}$ município $\mathrm{C}$, que admitiu que uma das principais vantagens é o acesso rápido às informações, neste item parece ter a maior noção do potencial da tecnologia. O município B relata que o sistema é multiusuário e o município D cita apenas o setor de cadastro e a diretoria do departamento de água e esgoto.

A maioria dos municípios usuários indicou que nível de satisfação não é bom. Apenas o município $B$ admite estar satisfeito com a utilização do sistema de informações geográficas. Dois municípios (C e D) mostram-se insatisfeitos e um (município A) não soube responder porque o sistema está inoperante.

As perspectivas para o futuro são boas. Todos os municípios manifestaram intenções de realizar melhorias.

Municípios não usuários de sistemas de informações geográficas

Nos 51 municípios que não adotam a tecnologia de sistemas de informações geográficas foi aplicado um questionário, que obteve 28 respostas.

Um dos objetivos desta etapa da pesquisa foi diagnosticar e avaliar a situação da representação dos traçados das redes de abastecimento de água e de esgoto sanitário em cada município.

Na Figura 2 observa-se que 68\% desses municípios ainda utilizam representações ana- 


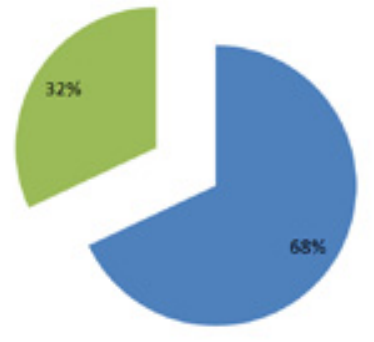

= Somente mapas

analógicos

= Mapas analógleos e

software CAD

n Somente software CAD

autros

ENSo ha representagles

Figura 2 - Formas de representação das redes de abastecimento de água.
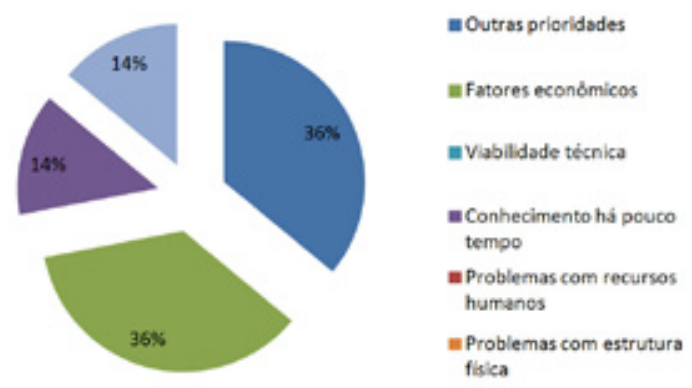

Figura 4 - Motivos de não utilizar sistemas de informações geográficas.

lógicas (mapa em papel) e 32\% usam uma associação de mapas em papel e em CAD para fazer a gestão das redes de abastecimento de água.

Considerando a gestão das redes de esgoto sanitário, nota-se na Figura 3 que 75\% desses municípios usam representações analógicas (mapa em papel) e 25\% usam uma associação de mapas em papel e em CAD.

De acordo com os resultados, é nítida a precariedade da situação, pois a grande maioria desses municípios utiliza somente mapas impressos em papel para gerir as redes de abastecimento de água e de esgoto sanitário.

Quanto ao conhecimento da tecnologia de sistemas de informações geográficas para gestão das redes, observou-se que 14 municípios a conhecem e 14 não. Isso mostra a desatualização do corpo de profissionais que trabalha nos órgãos gestores de saneamento urbano.

Considerando apenas os municípios que conhecem a tecnologia, buscou-se identificar justificativas para a não adoção dos sistemas

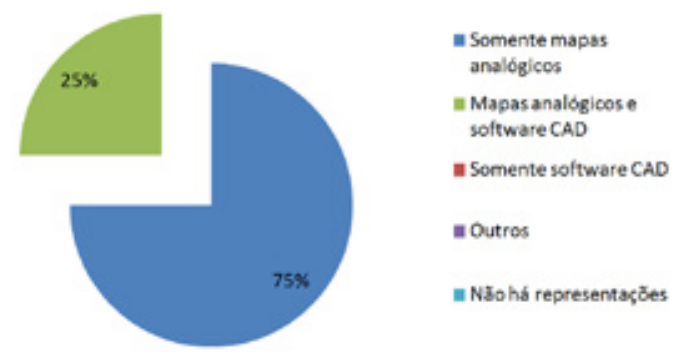

Figura 3 - Formas de representação das redes de esgoto sanitário.

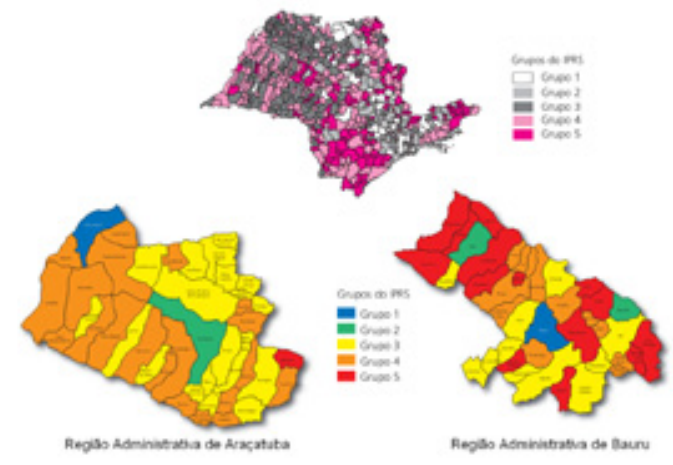

Fonte: SEADE (2010)

Figura 5 - Distribuição dos grupos do Índice de Responsabilidade Social Paulista.

de informações geográficas. As razões predominantemente apontadas foram: fatores econômicos e outras prioridades (Figura 4).

A resposta fator econômico (36\%) pode ser decorrente da falta de conhecimento das opções oferecidas pelos fornecedores desta tecnologia ou a dificuldade de encontrar soluções customizadas para as características do município. Observou-se que o município B conseguiu implantar uma solução de baixo custo e que tem atendido suas necessidades.

A resposta outras prioridades (36\%) pode dar um indicativo de que a viabilidade econômica já foi identificada e que há tendências favoráveis de crescimento do uso da tecnologia nas regiões administrativas.

Em toda a pesquisa não houve resposta quanto às estruturas física e recursos humanos (0\%), porém, contradizendo as respostas dos municípios usuários de sistemas de informações geográficas. Apesar do pequeno número 


\begin{tabular}{|c|c|c|c|c|c|c|c|}
\hline & & Área* & $\begin{array}{l}\text { \% da área de } \\
\text { São Paulo }\end{array}$ & População* & $\begin{array}{l}\text { \% da pop. } \\
\text { de São Paulo* }\end{array}$ & $\begin{array}{l}\text { Densidade } \\
\text { demográfica* }\end{array}$ & $\begin{array}{c}\text { Grau de } \\
\text { urbanização** }\end{array}$ \\
\hline & & $\left(\mathrm{km}^{2}\right)$ & (\%) & (Hab.) & (\%) & $\left(\mathrm{Hab} . / \mathrm{km}^{2}\right)$ & (\%) \\
\hline \multirow{2}{*}{$\begin{array}{c}\text { Regiões } \\
\text { Administrativas }\end{array}$} & Araçatuba & 18.562 & 7,5 & 740.350 & 1,78 & 39,88 & 92,13 \\
\hline & Bauru & 16.209 & 6,5 & 1.059 .963 & 2,55 & 65,39 & 94,38 \\
\hline Estado & São Paulo & 248.223 & 100 & 41.579 .695 & 100 & 167,51 & 95,94 \\
\hline
\end{tabular}

Quadro 2 - Características físicas e demográficas das Regiões Administrativas.

de implantações, também não houve resposta contra a viabilidade técnica ou econômica, ou seja, na região não houve nenhum questionamento ou impedimento quanto ao uso da tecnologia na gestão das redes de abastecimento de água e de esgoto sanitário.

\section{Conclusões}

O trabalho foi desenvolvido nas Regiões Administrativa de Bauru e de Araçatuba, ambas situadas no Estado de São Paulo. A Figura 5 mostra a distribuição dos grupos do Índice de Responsabilidade Social Paulista (SEADE, 2012), que variam entre 1 (melhor situação) e 5 (pior situação).

No Quadro 2 resumem-se as características físicas e demográficas das Regiões Administrativas de Araçatuba e de Bauru.

No Quadro 3 resumem-se alguns indicadores das Regiões Administrativas de Araçatuba e de Bauru.

Como se pode observar na Figura 5, em ambas as Regiões Administrativas a maioria dos municípios enquadram-se entre os grupos $3 \mathrm{e}$ 4 do Índice de Responsabilidade Social Paulista (SEADE, 2010). Esses resultados associados com os demais parâmetros mostrados nos Quadros 2 e 3 auxiliam no entendimento do perfil regional e dão subsídios para o entendimento dos resultados encontrados.

Considerando os municípios usuários de sistemas de informações geográficas, a implantação pioneira nas Regiões Administrativas de Bauru e de Araçatuba foi há cerca de 10 anos e a segunda há cerca de 7 anos. Atualmente existem somente mais duas implantações com a finalidade alvo deste estudo, somando $4 \mathrm{mu}$ nicípios usuários e incluindo 1 inoperante na época desses estudos. Isso caracteriza que o número de usuários é muito baixo e que não houve a evolução que se podia esperar na adesão à tecnologia, quando se considera seus benefícios decorrentes.

Uma constatação importante refere-se à estrutura do órgão gestor do saneamento urbano. Pelas entrevistas ficou nítido que na implantação de sistemas de informações geográficas os recursos humanos foram menos valorizados que os recursos físicos. A falta de capacitação e de quantidade insuficiente de funcionários foram os maiores problemas encontrados.

Os sistemas de informações geográficas parecem ser subutilizados, mas há tendência de institucionalizá-los em todos os municípios usuários da tecnologia.

As alterações da política municipal têm in-

\begin{tabular}{c|c|c|c|c|c|c|c|c|} 
& & \multicolumn{4}{|c|}{ IRPS* } & Abasteci- \\
mento \\
\end{tabular}

* 2010

Fonte: SEADE (2014)

Quadro 3 - Indicadores das Regiões Administrativas. 
fluenciado no desempenho dos sistemas de informações geográficas implantados. Houve interferência negativa em um dos municípios usuários da tecnologia. Além disso, as decisões políticas ainda foram citadas como motivo principal, segundo dois municípios, como causa do atraso tecnológico que se encontram. Com certeza este é um tema considerável para os órgãos gestores de saneamento urbano.

As receitas dos municípios não parecem ser impedimentos para a instalação de um sistema de informações geográficas. O município B, com menos de 10.000 habitantes, apresenta-se como uma solução satisfatória e com bom prognóstico.

Nas respostas dos questionários aplicados nos municípios não usuários de sistemas de informações geográficas, observou-se um elevado número de falta de conhecimento da tecnologia, e o restante das respostas admite o fator econômico e outras prioridades como os principais motivos. A resposta sobre o fator econômico parece estar associada à falta de conhecimento do mercado de fornecedores de softwares ou de encontrar soluções customizadas para o caso, como foi realizado no município B.

Nenhum município respondeu sobre a falta de estrutura física, porém, observou-se nos municípios usuários de sistemas de informações geográficas que os recursos humanos necessitam de atenção. Também não houve resposta sobre a falta de viabilidade, credenciando a tecnologia neste mercado.

Verificou-se também a precariedade das informações nos municípios não usuários de sistemas de informações geográficas, com dados desatualizados e mal gerenciados com mapas analógicos e também com pouco uso de software CAD. Observou-se que as redes de abastecimento de água apresentavam-se mais organizadas quando comparadas às redes de esgoto sanitário.

Para finalizar, é notória a falta de informação dos corpos técnicos e administrativos dos municípios das Regiões Administrativas estudadas. A maioria dos pesquisados citou problemas econômicos ou falta de conhecimento; entretanto, em contrapartida, o órgão do pequeno município B apresentou uma ótima implantação utilizando recursos próprios.
Referências bibliográficas

CÂMARA, G.; QUEIROZ, G.R. Arquitetura de sistemas de informação geográfica. In: CÂMARA. G.; DAVIS, C.; MONTEIRO, A.M.V. Introdução à Ciência da Geoinformação. Disponível em: < http:// www.dpi.inpe.br/gilberto/livro/introd/cap3-arquitetura.pdf>. Acesso em 10 Set. 2012.

DORCA, C.C; LUVIZOTTO JR, E.; ANDRADE, J.G.P. Aspectos da Implantação de um SIG em pequenos e médios Abastecimento de Água, UNICAMP - Universidade estadual de Campinas - Fac. Eng. Civil - DRH - Cidade Universitária "Zeferino Vaz" Campinas, 2001.

FERREIRA, C.D. Análise da Aplicação dos Sistemas de Informações Geográficas como Instrumento de Gestão dos Sistemas de Abastecimento de Água. Dissertação (Mestrado), UNICAMP - Universidade Estadual de Campinas, Faculdade de Engenharia Civil, Arquitetura e Urbanismo, Campinas - SP, 2005.

MARCONI, M.A.; LAKATOS, M.E. Metodologia do trabalho científico: procedimentos básicos, pesquisa bibliográfica, projetos e relatórios; publicações e trabalhos científicos. 6 ed. São Paulo: Atlas, 2007.

ROCHA, E.C.O.; VIEIRA, V.C.B.; CARNEIRO, E.L.N.C. Uso de Sistema de Informações Geográficas na atualização e modernização da área comercial da companhia de saneamento Águas e Esgotos do Piauí S/A. In: XIII Simpósio Brasileiro de Sensoriamento Remoto, Florianópolis, Brasil, 21-26 abril, INPE, p. 3081-3087. Anais. 2007.

SAIANI, C.C.S. Restrições à Expansão dos Investimentos em Saneamento Básico no Brasil: Déficit de Acesso e Desempenho dos Prestadores. Dissertação de mestrado - Faculdade de Administração, Economia e Contabilidade, Universidade de São Paulo, Ribeirão Preto, 2007.

SEADE. Fundação Sistema Estadual de Analise de Dados. O estado dos municípios 20062008. Índice Paulista de Responsabilidade Social. 2010. Disponível em: <http://www.seade.gov.br/projetos/iprs/ajuda/2008/sintese. pdf $>$. Acesso em 10 Set. 2012.

SEADE. Fundação Sistema Estadual de Analise de Dados. Informações dos municípios paulistas. Disponível em: <//http://www.seade.gov. br> . Acesso em 17 Fev. 2014. 